\title{
EREBEA
}

Revista de Humanidades

y Ciencias Sociales

Núм. 6 (2016), pp. 227-249

ISSN: 0214-0691

\section{Salvador de Madariaga y John B. Trend, catedráticos en OXFord y CAMBRIDGE: UN PUENTE CULTURAL ENTRE EsPAÑA Y REINO UNIDO}

\author{
Margarita Garbisu Buesa \\ Universidad a Distancia de Madrid, UDIMA
}

\section{RESUMEN}

La Universidad de Oxford creó su primera cátedra de Lengua y Literatura Españolas en 1927; la Universidad de Cambridge, seis años después, en 1933. Sus primeros ocupantes fueron el escritor español Salvador de Madariaga y el musicólogo e hispanista inglés John B. Trend, respectivamente. Antes de llegar a la cátedra ambos autores mantenían una estrecha relación con la cultura del país amigo, relación que la vida académica contribuyó a acrecentar. El presente artículo trata de ahondar en este vínculo y de mostrar la importante labor desempeñada por estos dos intelectuales como puente cultural entre Reino Unido y España durante las primeras décadas del siglo XX.

\section{ABSTRACT}

Oxford University created the first chair of Spanish Language and Literature in 1927; Cambridge University, six years later, in 1933. Their first occupants were, respectively, the Spanish writer Salvador de Madariaga and the English musicologist and hispanist John B. Trend. Before reaching the chair both authors maintained a close relationship with the culture of the friendly country, a relationship that academic life contributed to strengthen. This article tries to delve into this link and to show the important work carried out by these two intellectuals, acting as a cultural bridge between the United Kingdom and Spain during the first decades of the twentieth century.
Palabras Clave

Salvador de Madariaga; John B. Trend; cátedra de Español; Oxford; Cambridge.

\section{KeYwords}

Salvador de Madariaga; John B. Trend; Spanish Chair; Oxford; Cambridge. 

Con seis años de diferencia, las dos universidades más prestigiosas del Reino Unido crearon su primera cátedra de Lengua y Literatura Españolas: Oxford, en 1927 y Cambridge, en 1933. Curiosamente y al contrario de lo que cabría pensar, sus dos ocupantes iniciales no contaban con una formación en letras: fueron el español Salvador de Madariaga y el inglés John Brande Trend, el primero con estudios en Ingeniería de Minas, y el segundo, en Ciencias Naturales. Ambos nacieron en el último tercio del siglo XIX, en 1886, el primero, un año después, el segundo; ambos amaron sus respectivos países, pero pasaron largas temporadas fuera de ellos por trabajo, por devoción o por obligación; ambos llegaron a la cátedra sin experiencia docente previa, y a la literatura desde la divulgación o la ficción; ambos se vincularon con el entorno de la Institución Libre de Enseñanza y la Generación del 14, el español por pertenencia y el inglés por cercanía ideológica. Fueron dos melómanos confesos y, por ello, conocieron, admiraron y (en el caso de Trend) estudiaron la creación del maestro Manuel de Falla. Con Falla ambos coincidieron en Londres en mayo de 1921, cuando el músico viajó a la capital inglesa para participar en una serie de conciertos; Trend, que lo había conocido dos años antes en Granada, ejerció de anfitrión del compositor y, juntos, visitaron a Madariaga ${ }^{1}$.

En aquel entonces, aún faltaban años para que se convirtieran en catedráticos $y$, sin embargo, tanto uno como otro se habían sumergido en la cultura y la lengua del país amigo. Aunque resulte contradictorio, Madariaga se enamoró de Inglaterra en París, en los inicios de la década de los diez, y Trend llegó a España en 1919, con la intención previa de viajar a Italia. Pero aquellos hechos supusieron el principio del vínculo eterno de estos dos autores con las naciones que los adoptaron. Uno de los máximos exponentes de este vínculo fue la cátedra, si bien se manifestó igualmente en conferencias, libros, artículos, viajes, estancias y relaciones profesionales, personales e ideológicas, que convirtieron a las figuras de Salvador de Madariaga y John Brande Trend en puente entre Espańa e Inglaterra o en un hilo más de esa inmensa red de relaciones interculturales que se extendió en la Europa de entreguerras.

El objetivo del presente artículo es precisamente tratar de mostrar la aportación de estos dos autores como enlace del saber entre ambos países, incidiendo

1 M.J. Anstee, An Unlikely Spanish Don. The Life and Times of Professor John Brande Trend. Brighton, Portland, Toronto: Sussex Academic Press, 2013, p. 111. 
en su labor como catedráticos de Español en las dos universidades inglesas. Empezaremos por Oxford -pues Oxford fue la primera en la apuesta hispánica-, para seguir con Cambridge, aunque no sin antes recordar los antecedentes de las relaciones pedagógicas entre las dos culturas y lenguas. Madariaga y Trend llegaron a sus cátedras cuando el camino se encontraba algo - poco- andado. Ellos contribuyeron a marcarlo con fuerza, más Trend que Madariaga: la carrera docente del inglés se convirtió en su modus vivendi (además de en su vocación, su obsesión y su razón de ser) desde su nombramiento en 1933 hasta su jubilación un par de décadas después; en el caso del español, el ejercicio de la cátedra se limitó a algo más de dos años, pues la diplomacia y las relaciones internacionales llamaron insistentes a su puerta.

\section{Antes de las Cátedras de Oxford y Cambridge}

No es que hasta 1927, año en el que se creó la cátedra en Oxford, no se pudiera acceder a los estudios de Lengua y Literatura españolas en esta u otra universidad británica; era posible, pero no fácil. Oxford y Cambridge habían implantado estos estudios en el siglo XVIII, pero sin oficialidad alguna y más bien como "optativos, complementarios y hasta cierto punto con cierto carácter exótico”, en oposición a las lenguas clásicas, consideradas superiores ${ }^{2}$. No en vano estos dos prestigiosos centros se habían anclado en un tradicionalismo anglicano y un conservadurismo pedagógico que solo las jóvenes universidades surgidas en Londres a principios del siglo XIX pudieron contrarrestar; y estas, sí, incorporaron en sus programas oficiales los estudios de Lenguas Modernas, entre ellas, el Español. Así, tanto la Universidad de Londres (University College) como el King's College London, ambas nacidas entre 1826 y 1828, contaron en el siglo XIX con una cátedra de Español. En el primer caso, gozó de corta existencia (dos cursos académicos, de 1828 a 1830) y fue ocupada por Antonio Alcalá Galiano. En el caso del King's College, se instauró, para quedarse, en 1831, estableciendo una organización docente alrededor del catedrático, y con lectores e instructores como integrantes del departamento; su primer titular fue Pablo de Mendíbil, que ocupó el cargo durante tan solo doce meses.

Volviendo a Oxford y Cambridge, Gallardo Barbarroja explica que, en la primera, el estudio de Lenguas Modernas y sus literaturas no se introdujo de manera más o menos oficial "hasta bien entrada la segunda mitad del siglo XIX", aunque todavía no "como parte integral del plan de estudios, sino como asignaturas adicionales y optativas destinadas principalmente a los miembros de

2 M. Gallardo Barbarroja, "Introducción y desarrollo del español en el sistema universitario inglés durante el siglo XIX”, en Estudios de Lingüistica de Español (ELiES), vol. 20 (2003). Disponible en http://elies.rediris.es/elies.html. Consultado el 28/06/2016. 
la universidad que ya hubieran completado los estudios de grado o licenciatura”3. Con la creación en 1903 de la Escuela de Lenguas Modernas, por fin se incluyó oficialmente la enseñanza de Español, pero, a diferencia del King's, ni este ni ningún otro idioma contaba con cátedra individual propia.

En la Universidad de Cambridge la situación fue bastante más precaria. Las lenguas modernas se integraban en el departamento de Historia Moderna como materias complementarias. Gallardo Barbarroja constata una casi total "inexistencia de datos sobre el profesorado y las clases de Espańol" y duda de su existencia hasta la implantación de la cátedra en $1933^{4}$. Pero lo cierto es que sí se hallan trazas de la presencia del Español en Cambridge antes de ese año, si bien escasas y desequilibradas. Allison Sinclair confirma la inclusión en el claustro de lectores y profesores especiales en las primeras décadas de siglo, Villasante y Frederick A. Kirkpatrick, entre otros, a los que hay que añadir los significativos nombres de Pedro Salinas -lector en el curso 1922-1923- y Dámaso Alonso lector en los periodos 1923-1924 y 1928-1930-5 . A pesar de tan insignes docentes, siempre se consideró el Español como el pariente pobre de las lenguas modernas; era el Francés el que acaparaba casi toda la atención en el ámbito educativo.

La situación empezó a cambiar considerablemente después de la Primera Guerra Mundial, cuando en Inglaterra surgió un movimiento que tuvo como fin promover el desarrollo de la cultura española en tierras británicas. Como explica el profesor Ribagorda ${ }^{6}$, una de las primeras consecuencias de este movimiento fue la aparición de dos instituciones, con sedes en Londres y en Madrid: la AngloSpanish Society of Spanish-Speaking Countries and the British Empire, nacida en 1919, y el Comité Hispano-Inglés, nacido cuatro años después (si bien la aprobación de sus estatutos se fecha en mayo de 1926). La primera dependía del embajador español en Londres, que en aquel entonces era el marqués Alfonso Merry del Val y Zulueta; la segunda, de la Residencia de Estudiantes, pues a su amparo había nacido gracias al impulso de Alberto Jiménez Fraud y a la inestimable ayuda de Jacobo Fitz-James Stuart, el duque de Alba, y de Sir Esme Howard, entonces embajador británico en Madrid. Los objetivos de ambos organismos fueron similares: establecer un vínculo intelectual entre los dos países y promover la cultura del ajeno en el propio.

3 Gallardo Barbarroja, "Introducción y desarrollo...".

4 Gallardo Barbarroja, "Introducción y desarrollo...".

5 Villasante enseñó en Cambridge entre 1910 y 1917; Kirkpatrick, en el curso 1919-1920. A. Sinclair, "J.B. Trend en Cambridge. Comienzos y contexto", en Boletín de la Institución Libre de Enseñanza, no 89-90 (2013), p. 26.

6 A. Ribagorda, "El Comité Hispano-Inglés y la Sociedad de Cursos y Conferencias de la Residencia de Estudiantes (1923-1936)”, en Cuadernos de Historia Contemporánea, vol. 30 (2008), pp. 273-291. 
El Comité Hispano-Inglés significó un importante puente de unión entre España, por un lado, y Oxford y Cambridge, por otro, lo que no es de extrańar, ya que el ideario de la Residencia (y el de la Institución Libre de Enseñanza) se inspiró en los colleges de estas universidades. Precisamente uno de los logros del Comité fue la consolidación de un programa de becas destinadas a estudiantes españoles e ingleses, con el fin de cubrir estancias de seis meses en Oxford, Cambridge y Madrid, para la ampliación de estudios; fueron las llamadas "becas Howard", en honor al embajador.

Asimismo, entre los fines del Comité estuvo "la búsqueda de posibles mecenas para la creación de una cátedra de lengua española en Oxford", cátedra que, como sabemos, nació en 1927 bajo la denominación de The King Alfonso XIII Chair of Spanish Studies. A pesar de su nombre, los fondos para su instauración no provinieron del monarca, sino, en efecto, de una serie de mecenas, procedentes del mundo empresarial. Decía Salvador de Madariaga al respecto:

Pronto me enteré además de que ni Alfonso XIII ni el Gobierno español habían dado un céntimo para la cátedra, cuyo fondo se debía casi por entero a un chileno, magnate de la prensa, de origen y nombre judeo-galeses. La idea de ponerle corona a la cátedra se debía al promotor, que era un médico militar inglés llamado Bedford, que de español no sabía ni decir mañana, y cuyo fervor hispánico procedía de su deseo de ostentar la banda de una gran cruz-que consiguió. ${ }^{8}$

El magnate chileno al que Madariaga se refiere era Agustín Edwards MacClure, propietario del diario El Mercurio, a cuya contribución se unió la de William Morris (lord Nuffield), empresario del automóvil ${ }^{9}$. En julio de 1926 el propio rey Alfonso XIII inauguraba la cátedra durante un viaje a Oxford que realizó para ser investido doctor honoris causa por la insigne universidad. Un año después se iniciaron los trámites para su puesta en marcha, que terminó de consolidarse a principios de 1928, con la elección de titular. El nombramiento, que recayó en Salvador de Madariaga, se hizo oficial el 1 de enero de $1928^{10}$.

7 Ribagorda, “El Comité Hispano-Inglés y la Sociedad...”, p. 278.

8 S. Madariaga, Memorias (1921-1936). Amanecer sin mediodía. Madrid: Espasa-Calpe, 1974, p. 158.

9 Terra-EFE, "Colegio de Oxford quiere relanzar [el] lectorado que subsistió de Guillén a Marías", en Javiermariasblog, 1/11/2008. Disponible en: https://javiermariasblog.wordpress. com/2008/11/01/colegio-de-oxford-quiere-relanzar-lectorado-que-subsistio-de-guillena-marias/. Consultado el 28/06/2016.

10 O. Victoria, Vida de Salvador de Madariaga. Madrid: Fundación Ramón Areces, 1990, p. 88. 
Ya dijimos que Madariaga no tenía formación ni en lenguas ni en humanidades, con lo que la universidad le otorgó, por decreto, el título de Maestro de Artes para poder acceder al cargo $^{11}$. Y llega ahora el momento de responder a la pregunta de por qué: ¿por qué un Ingeniero de Minas, formado en París, acabó siendo catedrático de Lengua y Literatura españolas en una universidad británica? ¿De dónde provenía su vinculación con el Reino Unido? ¿Y de dónde su afición por las humanidades? El principio de las respuestas se puede hallar en una mujer y en los ferrocarriles, circunstancias vitales que tuvieron que ver con todo ello.

\section{Salvador de Madariaga, anglófilo y humanista}

Durante sus años universitarios en Francia, Madariaga se había relacionado muy estrechamente con un grupo de amigos oriundos de Gran Bretaña y Estados Unidos, que le llevaron a inmiscuirse de lleno en la lengua inglesa: "En mis años de Escuela Politécnica, y de Escuela Superior de Minas -recordaba Madariaga-, frecuentaba un grupo de jóvenes artistas ingleses y yanquis, y aun viviendo en París, hablaba más inglés que francés. De esta manera llegué pronto a manejar indistintamente las tres lenguas, a escribir prosa y verso en las tres y a hablar en público en las tres sin necesidad de texto escrito" ${ }^{12}$. Entre los amigos de este grupo, se encontraba la escocesa Constance Archibald, de la que Madariaga pronto se enamoró y que en 1912 se convirtió en su mujer.

Tal y como recordaba Nieves de Madariaga, la hija del matrimonio, ella le descubrió el misterioso mundo de su pais ${ }^{13}$. En 1910, el joven Salvador realizó un viaje a Inglaterra y Escocia que parecía anunciar una vida futura. Sin embargo, en 1911, ya como ingeniero de Minas, Madariaga se trasladó a Madrid para trabajar en la Compañía de Ferrocarriles del Norte de España: "Al dejar las minas por los ferrocarriles iniciaba ya sin darme cuenta mi evolución de lo técnico a lo humanista", sentenciaba ${ }^{14}$.

En efecto, permaneció en la capital hasta 1916 y durante esos años, aunque el interés literario le venía de lejos, se sumergió de lleno en la vida cultural del Ateneo; allí leyó a Miguel de Unamuno, coincidió con José Echegaray y escuchó por primera vez la música de Manuel de Falla, concretamente, sus Siete canciones españolas. También en esos años comenzaron los primeros contactos con el entorno de la Institución Libre de Enseñanza, con la generación del 14 (su generación) y con la política española: Jacobo Fitz-James Stuart, Américo Castro, Luis Araquistáin y José Ortega y Gasset y su Liga de Educación Política son algunos de los nombres que se relacionan en aquel entonces con la vida de Madariaga.

11 Victoria, Vida de Salvador..., p. 94.

12 Citado en Victoria, Vida de Salvador..., p. 10.

13 N. de Madariaga, "Sobre Salvador de Madariaga: paseos con mi padre”, en Cuenta y Razón, no 26 (1987), p. 9.

14 Citado en Victoria, Vida de Salvador..., p. 14. 
Fue precisamente Luis Araquistáin el causante de su marcha a Londres en 1916. Porque con el estallido de la Primera Guerra Mundial, John Walter, el dueño del Times, "había recibido el encargo del gobierno británico de organizar la propaganda inglesa" y necesitaba una persona para "dirigirla en todo el ámbito de lengua española”. ¿El elegido? Como se ha sugerido y por consejo de Araquistáin, Madariaga, que aceptó de inmediato la oferta, diciendo de este modo adiós a una prometedora carrera como ingeniero ${ }^{15}$ y saludando una nueva andadura profesional y vital: en 1916 la política internacional y la geografía inglesa entraban por primera vez de lleno en su vida.

En Londres, además de trabajar en la oficina de información sobre la guerra, escribía para diferentes medios españoles: para El Imparcial (del que era corresponsal), para España, con el pseudónimo "Julio Arceval", y para $L a$ Publicidad, periódico de Barcelona. Asimismo colaboraba con algunos medios ingleses: The Contemporary Review, The New Europe, The Observer... Sus artículos versaban sobre política pero también sobre literatura, pues en Londres su actividad cultural no solo no cesó sino que amplió miras: en la capital británica conoció a Ramiro de Maeztu, Ezra Pound o George B. Shaw, así como a Pablo Picasso, Sergei Diaghilev y a su admirado Manuel de Falla, a quienes en 1919 "trató personalmente durante las largas semanas de ensayos y preparativos que precedieron el estreno" de El sombrero de tres picos, el 22 de julio en el teatro Alhambra ${ }^{16}$. Como afirma Octavio Victoria, gracias a su intensa actividad, Madariaga pronto se convirtió en "el principal intérprete en España de los asuntos político-culturales del Reino Unido", así como "en el primer intérprete de los asuntos españoles en Gran Bretaña". También en 1919 pronunció la conferencia "Shelley and Calderón", que un año después quedó recogida en Shelley and Calderon and Other Essays on English and Spanish Poetry y le consagró como "pionero de los estudios anglo-españoles comparados"17.

En 1920 la estancia en Londres iba tocando a su fin ante la culminación de la guerra y el cierre de la oficina de información, carente entonces de sentido. Ese mismo año Madariaga realizó su primera incursión en el mundo académico al concursar para acceder a la cátedra de Español del King's College de Londres, que había quedado vacante tras el abandono de James Fitzmaurice-Kelly; no la logró, al parecer porque el embajador Merry del Val "vetó su candidatura"18. No importaba; en ese momento ganaba de nuevo en su vida la carrera internacional, pues en 1921 Madariaga se instalaba en Ginebra para trabajar en la Sociedad de Naciones, primero como secretario de la delegación española, después, como jefe

15 Victoria, Vida de Salvador..., p. 18.

16 Victoria, Vida de Salvador..., p. 38.

17 Victoria, Vida de Salvador..., pp. 42-44.

18 Victoria, Vida de Salvador..., p. 47. 
de la Sección del Desarme. En el país suizo permaneció hasta diciembre de 1927; meses antes, hastiado de su trabajo en la Sociedad de Naciones, había decidido presentarse de nuevo a una cátedra que, esta vez sí, siete años más tarde de su primer intento, conseguía sin problemas lo que provocaba su reencuentro con la geografía inglesa.

\section{MadARIAGA, CATEDRÁtico EN OXFord (I928-I93 I)}

Fue su gran amigo el hispanista Henry Thomas, quien le comunicó la convocatoria de la plaza. Explicaba Madariaga al respecto:

Thomas me escribió una carta a mano, recuerdo que en azul oscuro sobre azul claro. Me decía que se iba a crear en Oxford una cátedra de Lengua y Literatura Española y que él era vocal del Comité de Selección del profesor; y añadía que si yo le escribía a él una carta particular asegurándole que aceptaría, el Comité me ofrecería la cátedra. ${ }^{19}$

El sueldo sería de 1.110 libras, la mitad de lo que cobraba en Ginebra, pero Madariaga no lo dudó y se presentó a la convocatoria. Entre sus adversarios se podría haber encontrado John B. Trend, que se planteó competir por el puesto, si bien acabó desistiendo del empeño. Fue una decisión acertada ya que, como adelantamos, el 1 de enero de 1928 Salvador de Madariaga se convertía en catedrático en Oxford. Cinco meses después, el 15 de mayo, pronunciaba su conferencia inaugural, una disertación sobre la importancia del estudio de las lenguas y sobre el papel de la universidad como formadora de hombres sociales y no como meros eruditos del saber ${ }^{20}$. Como se ve, todo muy en la línea de las doctrinas pedagógicas de la época, que otorgaban a la educación un papel decisivo, huían de la excesiva especialización y abogaban por un saber humanista; los institucionistas, Ortega y Gasset, Pedro Salinas o T.S. Eliot fueron algunos de los intelectuales que defendieron el papel de la educación como pilar de la sociedad.

Según se deduce de las notas recogidas en el inventario de su archivo, Madariaga recorrió en sus clases las diferentes etapas de nuestra literatura: el Cid, los siglos XIV y XV, Fray Luis de León, el teatro del XVII, los narradores del XIX y, muy especialmente, Cervantes y el Quijote, así como los autores de la Generación del 98 (Ángel Ganivet, Azorín, Valle-Inclán, Pío Baroja y Unamuno) ${ }^{21}$. Es evidente

19 Madariaga, Memorias..., p. 158.

20 Victoria, Vida de Salvador..., p. 91.

21 Instituto "José Cornide" de Estudios Coruñeses, Cuadro de clasificación e inventario del fondo documental Salvador de Madariaga, caja 130. 
que el temario era amplio y cubría unas ambiciosas expectativas, pero no hay un buen programa sin un buen profesor que lo respalde, y este se convirtió en el mayor obstáculo para Madariaga: al contrario de lo que había podido imaginar, al nuevo catedrático no le gustaba su nueva profesión. La vocación de Madariaga no era ni la docencia ni la literatura como disciplina; lo suyo era la divulgación y las relaciones internacionales. Él mismo lo confesaba en sus Memorias:

Pronto me di cuenta de que las clases exigían de mi parte una gran energía intelectual (...) Así vine a pensar que daba a mis alumnos a la vez demasiado y no bastante, de modo que iba madurando en mí la idea que mi vocación no era aquella. ¿Cuál, pues? La mera contestación a esta pregunta revelaba otro de mis defectos como profesor. Mi verdadera vocación (...) era la de predicador de la buena nueva universal (...) mis pensamientos se orientaban hacia la situación internacional con más frecuencia y fuerza natural que hacia la cátedra y los estudiantes. ${ }^{22}$

Y lo cierto es que, en cuanto se le presentaba la ocasión, Madariaga se ofrecía para participar en círculos políticos y reuniones no universitarias, y dejaba de lado la labor docente que, en buena parte, descargaría en los profesores del departamento. En este sentido, contó con la inestimable ayuda del poeta Jorge Guillén, lector del departamento entre 1929 y $1931^{23}$. A pesar de ello, el catedrático no dejó pasar la oportunidad de abandonar las aulas durante unos meses cuando Federico de Onís, profesor entonces en la Universidad de Columbia en Nueva York, le propuso sustituirle en Oxford durante un año sabático: "acepté al instante"24.

En diciembre de 1930, Madariaga partía para Nueva York, México y Cuba, y, sin sospecharlo, comenzaba así un nuevo regreso a la carrera internacional. En tierras mejicanas le sorprendió la proclamación de la II República y en tierras cubanas, al desembarcar en la Habana el 1 de mayo de 1931, le sorprendió asimismo su nombramiento como embajador de la República de España en Washington; se enteró de él por la prensa, pero, como otras veces en su vida, aunque en esta ocasión tras mucho meditarlo, asumió el cargo ${ }^{25}$.

De este modo, Madariaga decía adiós a una corta carrera académica; como docente, ya hemos visto que él mismo reconoció que su labor en el aula no fue

22 Madariaga, Memorias..., p. 187.

23 Yo misma estudié la estancia de Jorge Guillén en la universidad inglesa en el artículo: "Jorge Guillén, lector en Oxford (1929-1931). El vínculo con Madariaga, Wilson y Eliot", en Analecta Malacitana, vol. XXXIV, no 1 (2011), pp. 79-98.

24 Madariaga, Memorias..., p. 228.

25 Victoria, Vida de Salvador..., pp. 124-125. 
brillante; pero como investigador, logró que la Oxford University Press lanzara "bajo los auspicios de la cátedra una serie de traducciones al inglés de clásicos sobre España"26; y como escritor, en sus años ingleses su obra se incrementó. De su época en Oxford son la novela Sir Bob (1930), o uno de sus ensayos más emblemáticos, Ingleses, Franceses, Españoles, que había redactado en su etapa ginebrina durante una convalecencia clínica y que se publicó entre 1928 y 1930 en los tres idiomas del título. Estas obras se unieron a su producción anterior, que era ya considerable: como traductor, había vertido al inglés algunas coplas españolas, bajo el título Spanish Folk Songs (1922); como crítico literario, había publicado, además del volumen sobre Calderón y Shelley, Semblanzas literarias contemporáneas (1923) o la Guía del lector del Quijote (1926), que dedicó a Manuel de Falla; y como creador, había gestado el poemario Romances de ciego (1922) o la novela La jirafa sagrada (1925). Además, había firmado numerosos artículos en medios españoles e ingleses, y había tratado con intelectuales, autores y políticos de ambos países. Todo ello le convertía en un referente indiscutible y puente de unión entre las dos culturas.

Por su parte, Jorge Guillén también abandonó Oxford en junio de 1931 para acceder a una cátedra en la Universidad de Sevilla. En Oxford había conocido al futuro gran hispanista Edward Meryon Wilson, que, en aquel entonces y tras haber pasado unos meses del curso 1929-30 en la Residencia de Estudiantes gracias a la concesión de una beca Howard, estudiaba y traducía la poesía de Luis de Góngora. Asimismo, días antes de su marcha, Guillén se había reunido con T.S. Eliot en Londres; en ese encuentro estuvo presente John B. Trend, que conocía al primero por sus viajes a España, y al segundo porque desde 1924 colaboraba como cronista musical con The Criterion, la revista que Eliot dirigía. Trend, por cierto, ante la renuncia de Madariaga, se presentó en 1931 -esta vez sí- a la cátedra de Oxford; sin éxito, pues el nuevo King Alfonso XIII Professor of Spanish Studies pasó a ser William J. Entwistle. Como explica Margaret Anstee, Trend se tomó con filosofía la "derrota" ${ }^{27}$. Su oportunidad llegaría dos años después.

\section{John B. TRend, Musicólogo e hispanista}

John B. Trend, como Madariaga, no contaba ni con experiencia docente previa ni con formación en humanidades o lenguas. Por consiguiente, cabe repetir la misma pregunta que nos planteamos con aquel: ¿por qué un licenciado en Ciencias Naturales por el Christ's College de Cambridge acabó siendo catedrático

26 Madariaga, Memorias..., p. 228. A decir verdad, se trató de volúmenes de historia más que de literatura: A History of Iberian Civilization, de Oliveira Martins, y Liberty and Despotism in Spanish America, de Cecil Jane.

27 M. J. Anstee, “J.B., un académico errante del siglo XX”, en Boletín de la Institución Libre de Enseñanza, no 89-90 (2013), p. 40. 
de Lengua y Literatura españolas? ¿De dónde provenía su vinculación con España? ¿Y de dónde su afición por las humanidades? Si en el caso de Madariaga hablamos de Constance Archibald, su mujer, como una de la causas de su amor por Inglaterra, en el caso de John B. Trend debemos aludir a Edward J. Dent, musicólogo y profesor en Cambridge, como figura clave en su formación e, indirectamente, en su amor por España.

A decir verdad, y a pesar de su educación científica, Trend se sintió desde su juventud muy atraído por las doctrinas humanísticas, especialmente por la música. En sus años universitarios, dirigió su trayectoria hacia la divulgación cultural, ya que fue "corresponsal del Christ's College en la Cambridge Review, editor de la Christ's Magazine y publicaba artículos en distintas revistas" ${ }^{28}$. Fue esta afición la que le llevó a vincularse desde 1908 con Dent y su círculo, un grupo de brillantes intelectuales, socialistas, anticlericales y antivictorianos, que hicieron mella en un hasta entonces conservador y religioso Trend. El pensamiento y la ideología del universitario comenzaron a cambiar, y su amor por la música y la divulgación, a incrementarse; "Dent taught him the techniques of musical analysis and criticism and encouraged his enthusiasm", explica Edward M. Wilson ${ }^{29}$.

Trend luchó en la Primera Guerra Mundial, una experiencia traumática en su persona, por lo que, a su regreso del frente, le faltó tiempo para volver a su antigua vida de música y periodismo. Viajero impenitente, quiso trasladarse a Italia para indagar en su herencia cultural, pero Dent le convenció para que se decantara por España, un país desconocido y con un valioso legado musical por explorar. Trend no lo dudó; gracias también a Dent, consiguió colaboraciones en diversas revistas de la época, "including John Middleton Murry's Athenaneum, which was one of the most prominent" ${ }^{30}$, y, como corresponsal de The Athenaeum, llegó en 1919 a España. Y en España, el joven periodista halló un país mucho más sorprendente de lo que intuía.

Porque Trend no sospechaba que se iba a encontrar en Madrid con el ambiente cultural de la Residencia de Estudiantes; ni sospechaba que iba a tratar de cerca a las figuras artísticas más destacables del país; ni que descubriría tesoros musicales en su búsqueda investigadora. Sin embargo, así sucedió desde su primer contacto con la Península. Como explica James Valander, en octubre de 1919 Trend conoció a Alberto Jiménez Fraud en una visita que realizó a la Residencia y "parece que desde un principio los dos hombres se entendieron a la perfección" ${ }^{1}$. En Granada, en ese mismo año conoció a Manuel de Falla primero y a Federico

28 Anstee, “J.B., un académico...”, p. 33.

29 E.M. Wilson, "John Brande Trend. 1887-1958”, en Bulletin of Hispanic Studies, vol. XXXV (1958), p. 223.

30 Anstee, An Unlikely Spanish Don..., p. 91

31 J. Valender, "El profesor J.B. Trend y Alberto Jiménez Fraud: de la guerra civil al exilio", en Boletín de la Institución Libre de Enseñanza, no 89-90 (2013), p. 160. 
García Lorca después. El propio Trend recordaba estos encuentros: "The first time I met Don Manuel de Falla was on a blustering September evening at the Villa Carmona on the Alhambra Hill" ${ }^{2}$, decía sobre el músico. Al poeta le escuchó recitar unos versos en un jardín del Albaicín y se quedó prendado: "Who is it?", preguntó: "Federico García Lorca. You must meet him"33. Ese día la velada terminó a las cuatro de la madrugada con Lorca y Trend dando tumbos por las estrechas calles granadinas.

Trend en seguida se sintió cautivado por España; aprendió bien el idioma y sus viajes por nuestro país no cesaron en los años veinte ${ }^{34}$. Su actividad, en principio, se volcó en la música: recorrió diferentes bibliotecas para explorar antiguas partituras, estudió las melodías renacentistas españolas de creadores como Tomás Luis de Victoria o Cristóbal de Morales y aprendió igualmente a entender la modernidad del maestro Falla. Ello, sin embargo, no fue óbice para que se involucrara de lleno en el ambiente madrileño de la Residencia y se relacionara, además de con Jiménez Fraud, con otros institucionistas como José Castillejo, así como con los autores del 98, los intelectuales del 14 y los jóvenes poetas del 27. Él mismo lo explicaba de esta manera:

In the early nineteen-twenties I had time in Spain to 'sit about' and listen to people talking: Juan Ramón Jiménez, Unamuno, Pío Baroja, Ortega Gasset, Enrique de Mesa, Pérez de Ayala, Antonio and Manuel Machado, Moreno Villa, Amado and Dámaso Alonso, Pedro Salinas, Jorge Guillén, Vicente Aleixandre, García Lorca, Rafael Alberti, Luis Cernuda, the Mexican Alfonso Reyes and the Spanish composer Manuel de Falla. ${ }^{35}$

Como buen divulgador, Trend quiso transmitir en su país sus descubrimientos hispánicos (tanto musicales como literarios, intelectuales y humanos), y sobre ellos escribió en artículos y libros. Así, además de en The Athenaeum, abordó la cultura de nuestro país (sobre todo su música) en Music and Letters, The Times Literary Supplement o The Criterion; e igualmente en los volúmenes A Picture of

32 J.B. Trend, A Picture of Modern Spain: Men \& Music. London: Constable \& Company, 1921, p. 237.

33 J.B. Trend, Lorca and the Spanish Poetic Tradition. New York: Russell \& Russell, 1971, p. 2.

34 Tess Knighton ha reconstruido las fechas de sus visitas a España gracias a sus escritos y su correspondencia. Entre los años 1919 y 1930, Trend viajó cada año (excepto en 1924) por diferentes provincias espańolas. Sus estancias más largas fueron en 1919 (entre julio y diciembre), en 1920 (entre mayo y septiembre), en 1921 (entre julio y noviembre), en 1923 (entre marzo y julio) y en 1926 (entre enero y julio). Véase T. Knighton, "John Brande Trend (1887-1958) and His Musical Iter Hispanicum", en Music and Letters, no 19 (2014), p. 28.

35 Trend, Lorca and the Spanish..., p. 172. 
Modern Spain: Men and Music (1921), Luis Milan and the Vihuelistas (1925), The Music of Spanish History to 1600 (1926), Alfonso de Sage and other Essays (1926) o Manuel de Falla and Spanish Music (1929) ${ }^{36}$. Y fuera cual fuera el argumento concreto de su escrito, Trend siempre perseguía una misma intención: como bien afirma Nigel Dennis, "hacer de puente o intermediario entre Espańa e Inglaterra, explicando al público británico el sentido de la cultura española, en su dimensión histórica y actual", un público "curioso pero mal informado" que no veía más allá de leyendas románticas y estereotipos culturales ${ }^{37}$, esto es, que identificaba el nuestro con un país bárbaro, de gitanas, flamenco y matadores.

Para conseguir su objetivo, Trend se valió en sus textos de dos procedimientos: por un lado, denunció directamente el estereotipo mostrando a sus lectores una España plural y variada, alejada de esa visión única que sus paisanos habían grabado en sus mentes; y, por otro, dio a conocer en Inglaterra el europeísmo de la cultura hispánica. En este sentido, en el capítulo inicial de A Picture of Modern Spain. Men and Music, titulado "Spain after the War", Trend afirmaba:

Travel and conversation with men of very different classes in very different provinces shows convincingly that the European view of life and its values -that mixture of idealism, humanity and common sense with other ingredients not so easily described- is more alive today in Spain than in most other countries. Listening to Spaniards I have often felt that they are expressing a point of view which is very 'English'. ${ }^{38}$

Y afirmaba asimismo que ese europeísmo se debía, en muy buena medida, a la labor educativa realizada por sus amigos Jiménez Fraud y Castillejo, así como por Francisco Giner de los Ríos, mediante la Institución Libre de Enseñanza, la Junta para Ampliación de Estudios y la Residencia de Estudiantes. Estos intelectuales -explicaba- trataron de alfabetizar España y paliar la nefasta influencia que la Iglesia había ejercido sobre el pueblo durante años. Trend, que -al igual que

36 Manuel de Falla and Spanish Music es la primera monografía publicada sobre el compositor gaditano. La relación entre Trend y Falla fue profunda tanto profesional como personalmente. Además de escribir artículos y libros sobre su música, Trend la dio también a conocer en Inglaterra a través de conciertos y traducciones. A cargo de Trend estuvo, por ejemplo, el estreno en Bristol en 1924 del Retablo de Maese Pedro, así como la traducción del propio Retablo o del "Soneto a Córdoba”, de Góngora, al que Falla puso música dentro de las conmemoraciones del tercer centenario de la muerte del poeta cordobés. Asimismo, la correspondencia entre los dos amigos (editada por Nigel Dennis en 2007) dan fe de la confianza existente entre ellos. Véase N. Dennis (ed.), Manuel de Falla - J.B. Trend. Epistolario (1919-1935). Granada: Universidad de Granada. Archivo Manuel de Falla, 2007.

37 N. Dennis, "Prólogo" en Dennis (ed.): Manuel de Falla - J.B. Trend..., p. 10.

38 Trend, A Picture of Modern..., p. 1. 
Madariaga- "consideraba la educación (...) como uno de los elementos cruciales para el desarrollo de un país" 39 , comprendió que el estamento religioso había coartado ese desarrollo en España al haber supeditado la formación de las gentes a la fe católica. Si el círculo de Dent había contagiado a Trend un agnosticismo inicial, el descubrimiento de semejante verdad consiguió alejarle definitivamente de la iglesia.

Trend también mostró su admiración por la labor institucionista en "Education in Spain", otro capítulo de A Picture of Modern Spain: Men and Music, que además se adecua perfectamente al discurso de este trabajo. Porque en él Trend comparó la Residencia de Estudiantes con los colleges de Cambridge y Oxford: "The Residencia is like (...) certain colleges at Oxford and Cambridge -afirmaba-, because of the friendship and social intercourse which exists between the junior and more senior members of it, between undergraduates and dons" 40 . El amor que profesaba a la Residencia le llevó a participar en sus tertulias y actividades (sobra decir que en ella se alojaba en sus visitas a Madrid) y a colaborar muy estrechamente con su Comité Hispano-Inglés, al que previamente nos hemos referido. Entre otras muchas tareas, en el año 1926 ejerció de anfitrión de John Maynard Keynes, el general Charles Bruce, H.G. Wells o Gilbert Keith Chesterton, en sus estancias en la capital española como conferenciantes ${ }^{41}$. Asimismo Trend firmó diversos artículos sobre la cultura de su país en Residencia, la revista de la institución.

En definitiva, al igual que Madariaga, Trend se convirtió por méritos propios en un referente indiscutible y en puente cultural entre Inglaterra y nuestro país. Y, al igual que Madariaga y a pesar de no contar con la formación académica pertinente, estaba totalmente legitimado para presentarse en 1933 a la cátedra de Lengua y Literatura españolas en la Universidad de Cambridge.

\section{TRend, CATEdrático en CAMbridge (I933-I953)}

Si en 1927 Trend no se animó a concursar por la cátedra de Oxford fue por su claro anticlericalismo y, más concretamente, por su oposición a los jesuitas. En aquel momento Primo de Rivera regía España y la sombra de la iglesia era tan alargada que podía llegar hasta Inglaterra y vetar a un descreído como él. Ya comentamos que en 1931 lo intentó por primera vez, pero la II República aún era joven y Entwistle se ajustaba perfectamente a la ortodoxia exigida por Oxford. Sin embargo, en 1933, cuando la Universidad de Cambridge creó su Chair in Spanish Studies, Primo de Rivera y la monarquía habían pasado a buen recuerdo, la iglesia había dejado de ser poderosa y la República se había consolidado, para inmensa

39 Á. Ribagorda, “J.B. Trend: Un hispanista inglés en la Residencia de Estudiantes”, en Boletín de la Institución Libre de Enseñanza, no 89-90 (2013), p. 120.

40 Trend, A Picture of Modern..., p. 37.

41 Anstee, An Unlikely Spanish Don..., p. 132. 
alegría de Trend, que se había convertido en uno de sus más férreos defensores. Así las cosas, esta vez Trend lo intentó de nuevo y en un disputado concurso que le acarreó una eterna rivalidad con el hispanista Allison Peers, logró la plaza. "Pero he tenido una infinidad de cartas y muchísimas preocupaciones, entre ellas, el (sic) de salir elegido catedrático en la universidad de Cambridge- le explicaba a Manuel de Falla en una carta fechada en Londres el 22 de septiembre de ese año-. ¡Figúrese! ¡Cosa tan inesperada! $Y$ tan agradable, teniendo tantos amigos alli’”‘2.

Desde ese momento, Trend se volcó en su nueva profesión: había que levantar un departamento muy abandonado (con goteras en el tejado y "material escolar malo", según le contaba a Falla ${ }^{43}$ ), reorganizar el claustro de profesores y decantarse por una línea pedagógica que seguir. $Y$ es que antes de la llegada del nuevo catedrático, en la caótica enseñanza del Español existían dos corrientes distintas: una reflejaba "un concepto bastante tradicional del currículo, no del todo ajeno a la idea de 'grandes obras' (Cervantes, Calderón etc.)”, mientras que en la otra se podía "discernir no solo variedad, sino incluso excentricidad en lo que se impartía” 44 .

Sin duda, era necesario unificar criterios, y en esto Trend tuvo meridianamente claro que iba a continuar la estela de la Institución Libre de Enseñanza y la Residencia de Estudiantes, de igual modo que años atrás Giner, Castillejo y Jiménez Fraud habían continuado la de los colleges de Oxford y Cambridge. De este modo, con la aplicación del proyecto institucionista, Trend quiso "propiciar una revolución -un renacimiento- en el enfoque de la enseñanza del español” ${ }^{45}$, y alejarse así de la postura docente oficial y oficialista de las universidades británicas.

Para ello, una de sus primeras medidas fue contratar como profesor ayudante a Edward M. Wilson que, como sabemos, conocía sobradamente la línea pedagógica de la Residencia pues en ella había vivido durante unos meses del curso 1929-30. Asimismo, aunque Trend incluyó en sus clases a autores como Feijoo, Cadalso o Quintana, optó por dedicar buena parte del programa docente "a explicar la historia de la Institución Libre de Enseñanza y su decisiva influencia en la modernización del país"46. Alumnos de Trend fueron el hispanista Nigel Glendinnig o la propia Margaret Ansteee, su biógrafa, quien recuerda que, desde un punto de vista tradicional, Trend no destacaba como el mejor de los profesores, si bien transmitía entusiasmo por España y propiciaba el diálogo y la interacción con sus estudiantes ${ }^{47}$.

42 Dennis (ed.), Manuel de Falla - J.B. Trend..., pp. 215-216.

43 En la carta citada. Dennis (ed.), Manuel de Falla - J.B. Trend..., p. 215.

44 Sinclair, "J.B. Trend en Cambridge...", p. 26.

45 Anstee, "J.B., un académico...", p. 40.

46 Ribagorda, “J.B. Trend: un hispanista inglés...”, p 136.

47 Anstee, An Unlikely Spanish Don..., p. 150. 
En su labor pedagógica, Trend también comprendió la necesidad de relacionar la literatura con otras disciplinas humanísticas dentro de un todo cultural. Comprendió -y aquí fue pionero- el valor y el peso de Latinoamerica en la cultura hispánica: "JB was one of the very first Hispanists to recognise the importance of Latin American and to extend Spanish Studies to cover that region", afirma Anstee ${ }^{48}$. Comprendió la validez de contar con buenos docentes, motivo por el cual ficharía a la hispanista Helen Grant o respaldaría el nombramiento de lectores de la talla de Jesús Bal y Gay (entre 1935 y 1937), José Antonio Muñoz Rojas (1937-39), Enrique Moreno (1939-41), Esteban Salazar y Chapela (1941-43) o Luis Cernuda (1943-45), aunque este último resultó ser un profesor nefasto ${ }^{49}$. Y comprendió igualmente la riqueza del testimonio directo, lo que le llevó a invitar a la universidad a intelectuales como Miguel de Unamuno, que acudió a Cambridge en febrero de 1936. Muñoz Rojas recordaba el encuentro con el escritor de este modo:

Don Miguel llegó a Cambridge en febrero de 1936. Creo que venía a dar unas conferencias en la Spanish Society que no dio. En cambio, le reunió el profesor Trend con los estudiantes de español y algunos españoles que por allí andaban. (...) Llegó don Miguel y se sentó campechanamente entre nosotros. (...) El profesor Trend, que era hombre tartamudeante a la inglesa, nervioso, lo introdujo con unas palabras breves. ${ }^{50}$

Unos meses después de la visita de Unamuno estallaba la guerra civil en España y, como no podía ser de otro modo, el enfrentamiento político del país se trasladó también a Cambridge. Frente a un republicano convencido como Trend, otros profesores, como Theodore Bullock, defendían el levantamiento de Franco; la tensión era más que evidente y las fisuras ideológicas acabaron marcando las actividades del departamento ${ }^{51}$.

A pesar del gran esfuerzo que le suponía la gestión académica y la labor docente, Trend no descuidó sus escritos durante su etapa como catedrático, si bien estos entonces se centraron en las letras no tanto así, como en la década previa, en la música. Entre otros, como editor y traductor, publicó Prosa diversa, de Miguel de Unamuno (1938), Prose and Verse, del Marqués de Santillana (1940) o Fifty Spanish Poems, de Juan Ramón Jiménez (1950); y como ensayista, "Calderón and the Spanish Religious Theatre of the Seventeenth Century" (1938), Antonio

48 Anstee, An Unlikely Spanish Don..., p. 157.

49 Anstee, “J. B., un académico...", p. 44.

50 J.A. Muñoz Rojas, Amigos y maestros. Valencia: Pre-Textos, 1992, p. 41.

51 Anstee, “J. B., un académico"..., p. 42. 
Machado (1953), o la serie de separatas publicadas por su universidad, dedicadas a Jorge Guillén, Federico García Lorca, Rubén Darío, Gonzalo de Berceo y San Juan de la Cruz, que quedarían después recogidas en Lorca and the Spanish Poetic Tradition (1956).

Como se puede comprobar, la atención de Trend se centró preferentemente en la literatura antigua o en los autores de la Edad de Plata, la época cultural que había vivido y de la que había formado parte en los años veinte. Paradójicamente, en los años treinta, sus viajes por España disminuyeron, pero por dos claros motivos: la ingente cantidad de trabajo que acarreaba la cátedra y, por su puesto, la inestabilidad política del momento. Trend se trasladó a España por última vez -aunque esto entonces él no lo sabía- en plena contienda; en concreto, visitó Valencia en el verano de 1937. Es fácil pensar que la razón del desplazamiento fue la asistencia al II Congreso Internacional de Escritores Antifascistas, que se celebró en el mes de julio entre Valencia, Madrid y Barcelona; sin embargo, Anstee considera que lo que lo motivó tuvo que ver con "medidas de urgencia para salvar las grandes obras de arte del Prado y otros museos del intenso bombardeo a que estaba siendo sometido Madrid" 52 .

Después de este viaje Trend se negó a pisar de nuevo suelo español mientras Franco se mantuviera en el poder. Un republicano como él no podía comulgar con la dictadura.

\section{El EXILIO}

En resumidas cuentas, y aunque por razones obvias el término no sea el apropiado, Trend se exilió de España por propia iniciativa. Sin embargo, no permaneció callado y mostró su desacuerdo con el régimen mediante la escritura o mediante los propios hechos.

Por ello, en el Times Literary Supplement del 17 de octubre de 1936 publicó una sentida carta de condena por el asesinato de Federico García Lorca, que remataba con estas palabras:

The murder took place between August 10 and 15. It was useless to intercede with the rebel 'authorities' and the people of Granada had the privilege of seeing a poet's books publicly burnt in the Plaza de Carmen, as a new auto da fe. The circumstances of the arrest, the trumped-up charge, and the barbarous detail of the burning of the books of verse show what the attitude of military-clerical reaction is likely to be towards literature and art. The least an English friend can do is to make these things public..$^{53}$

52 Anstee, “J.B., un académico...", p. 43.

53 Citado en Anstee, An Unlikely Spanish Don..., pp. 166-167. 
Trend perdió igualmente a Falla, que se exilió en Argentina en 1939; y como a estos dos amigos, a tantos otros. Y por ello $-\mathrm{y}$ aquí se ve su denuncia mediante hechos-, no dudó en ayudar a españoles que huyeron de Franco (ciudadanos célebres o anónimos), facilitándoles asilo y trabajo en su país: al músico Roberto Gerhard, a quien le consiguió un puesto en el King's College London, a Antonio Machado, que murió antes de conocer la noticia de su nuevo destino en Cambridge ${ }^{54}$, o a un nutrido grupo de niños vascos. Con otros como Jorge Guillén, no lo consiguió: el poeta acudió a él solicitándole un lectorado en su universidad, un puesto que Trend no le pudo facilitar al estar ya ocupado por Muñoz Rojas ${ }^{55}$.

A este elenco se unió Alberto Jiménez Fraud, lo que no resulta en absoluto sorprendente ya que este nombre simbolizaba la España de Trend, la España educada, culta, abierta, laica y europea que había conocido en los años veinte, la de la Residencia y la Edad de Plata: una España que ahora, con el dictador, podía desaparecer. De ahí que, como decíamos, no resulte sorprendente que, en octubre de 1936, Jiménez Fraud y su familia se trasladaran a Cambridge gracias a la hospitalidad del catedrático. El propio Jiménez Fraud se refería a este episodio de la siguiente manera:

A mediados de octubre salíamos mi mujer y yo para Cambridge, acompañados de Natalia y de mi hijo (...) En Cambridge nos esperaba el hispanista J.B. Trend, quien me ofreció las conferencias Norman-MacColl que cada cuatro ańos se dictan en aquella universidad y que son un honor apetecido. ${ }^{56}$

Pero las Norman-MacColl no eran más que una solución provisional, por lo que, en 1937, Entwistle, el sucesor de Madariaga en la cátedra, invitó a Jiménez Fraud a dictar unas nuevas conferencias en su universidad. Así las cosas, el intelectual se acabó trasladando a Oxford en el otoño de 1938 para convertirse en lector de Español: "La duración del puesto nuevo era por un máximo de tres años -explica Valender-; sin embargo, el contrato fue renovado y Oxford, de

54 El profesor Nigel Dennis, en "Asilo en Cambridge para Antonio Machado", sacó a la luz este episodio. En este artículo se reproduce una carta del 24 de febrero de 1939, que José Machado envió a Trend agradeciéndole el ofrecimiento y comunicándole que su hermano Antonio acababa de morir, por lo que nunca llegó a conocer la propuesta. Véase N. Dennis, "Asilo en Cambridge para Antonio Machado (Un ofrecimiento del Profesor J.B. Trend con una carta inédita de José Machado)", en Cuadernos Hispanoamericanos, vol. 304-307 (1975-1976), pp. 445-449.

55 Varias cartas entre Trend y Guillén de 1937 y 1938, dan fe de ello. Yo misma las analicé en el artículo: "Jorge Guillén y John B. Trend, un amistad marcada por el exilio", en Bulletin of Spanish Studies, vol. 89.3 (2012), pp. 391-414.

56 Alberto Jiménez Fraud, Residentes. Semblanzas y recuerdos. Madrid: Alianza, 1989, p. 34. 
hecho, se convirtió en el permanente lugar de residencia de don Alberto, no solo hasta su retiro de la universidad en 1953, sino más o menos hasta el final de su vida en $1964 " 57$.

Precisamente otro de los miembros de la colonia de exiliados en la ciudad inglesa fue Salvador de Madariaga. El antiguo catedrático se instaló en Oxford también en 1938. Había abandonado España el 31 de julio de 1936: MadridValencia-Barcelona-Port Bou-Perpiñán, según él mismo lo relataba en sus Memorias $^{5}$. La guerra le había sorprendido en Toledo y en seguida tuvo claro que debía salir del país. En este sentido, recordemos que había dejado la cátedra de Oxford en 1931 para ser nombrado embajador de España en Estados Unidos. Pero su servicio a la República no se había quedado ahí: también en 1931 fue delegado de Espańa en la Sociedad de Naciones y en enero de 1932, embajador de España en París ${ }^{59}$. Asimismo, lejos de la política internacional, en 1934 se había convertido en ministro de Instrucción Pública y Bellas Artes, si bien su paso por el gobierno no superó los dos meses. Por sus contactos ministeriales, a finales de ese año conoció en persona a Francisco Franco, que entonces era Jefe del Estado Mayor; le llamó la atención su "inteligencia concreta" y su falta de ostentación ${ }^{60}$. Después, cuando subió al poder, pidió públicamente al tirano que se marchara y, como Trend, renunció a pisar Espańa mientras el país se mantuviera bajo su mando.

Por todo lo dicho, en 1938 Madariaga regresó a su querida Oxford, aunque no recuperó su antigua labor docente. Desde allí no cesó de viajar por el mundo dando entrevistas y conferencias, y luchando por la defensa de una Europa unida (a este respecto, uno de sus mayores legados fue la creación en 1949 del Colegio de Europa en Brujas), y continuó con su labor como escritor. Entre su creación en el exilio, destacan varios volúmenes dedicados a la historia del Imperio Español en América. Como ya adelantamos, Madariaga había viajado en 1931 por México y Cuba; en el verano de 1935 volvió a tierras americanas en un tour que le llevó por Argentina, Chile, Perú y Brasil. Aun siendo un europeísta convencido, Madariaga adoraba Latinoamérica (México sobre todo) y, por la admiración y curiosidad que le despertaba aquel continente y su historia, en 1939 publicó en Londres Columbus, en 1942, también en Londres, Hernán Cortés (que saldría un año después, en castellano, en Buenos Aires), y en 1951, Bolivar en Méjico ${ }^{61}$.

57 Valender, “El profesor J.B. Trend y Alberto Jiménez Fraud...”, p. 164.

58 Madariaga, Memorias... pp. 562-563.

59 Para conocer la labor internacional de Madariaga durante la II República, véase M.R. de Madariaga Álvarez-Prida, "Salvador de Madariaga y la política exterior española durante la II República”, en RIPS. Revista de Investigaciones Politicas y Sociológicas, vol. 8, no 2 (2009), pp. 85-95.

60 Victoria, Vida de Salvador de Madariaga..., p. 196.

61 A. López Prado, Sintesis biográfica de don Salvador de Madariaga. La Coruña: Instituto José Cornide de Estudios Coruñeses, 1993, p. 10. 
Justamente, cuatro años antes, en 1946, John Trend había sacado Bolivar and the Independence of Spanish America, atraído, como Madariaga, por el libertador y, en general, por el continente latinoamericano, que había pisado por primera vez en las navidades de 1937-38, en una visita a Cuba junto con Dent. Durante las navidades siguientes conoció México, país que, como España dos décadas atrás, le cautivó de inmediato: "He fell in love with the country from the moment he set foot on Mexican soil" ${ }^{2}$. El motivo del desplazamiento fue, entre otros, visitar a los amigos españoles allí exiliados y acogidos en la Casa de España, como José Moreno Villa, Jesús Bal y Gay, o el mejicano Alfonso Reyes. Regresó pocos meses después, en abril de 1939 y, fruto de estos viajes, publicó en 1940 Mexico: A New Spain with Old Friends, un volumen con su personal visión de los lugares y las costumbres de aquellas tierras, que, sin embargo, contiene una fuerte carga de nostalgia hacia España. Afirmaba Trend en el capítulo introductorio:

This invitation by a 'Spanish House' to a number of the best representatives of that Spain which can never be destroyed, the Spain which is a country of the mind; and it is a lesson which Spaniards -all good Spaniards- will take to heart. Spain is not done for; she will go on and begin again in Spanish America. ${ }^{63}$

Una vez más, Trend reivindicaba la España de su memoria, que entonces se congregaba al otro lado del Atlántico y que, dado que América estaba demasiado lejos, intentó hallar en Portugal. Como explica Anstee, después la guerra mundial, los viajes de Trend se limitaron a Europa y, especialmente, a Portugal; aprendió portugués y allí "buscó el encanto que había encontrado en España"64.

Cumplió su promesa de no visitarla mientras Franco permaneciera en el poder; y por este motivo no pudo ver de nuevo, después de 1937, su país de adopción. El 20 de abril de 1958 Trend fallecía, inesperadamente, en Cambridge. Cinco años antes se había jubilado; le sucedió en la cátedra Edward Meryon Wilson, su ayudante en 1933, catedrático en Londres desde 1945 y, en 1953, hispanista de prestigio.

\section{A MODO DE CONCLUSIÓN}

De Edward Wilson fue la iniciativa de crear una beca en memoria de Trend para que los estudiantes de Cambridge pudieran "con mayor facilidad hacer viajes de estudios por España, Portugal y quizá otros países de lengua española

62 Anstee, An Unlikely Spanish Don..., pp. 183-184.

63 J.B. Trend, Mexico. A New Spain with Old Friends. Cambridge: Cambridge University Press, 1941 , p. 3

64 Anstee, “J.B., un académico...”, p. 48. 
o portuguesa”, tal y como le explicaba a Jorge Guillén en una carta del 24 de octubre de $1958^{65}$. En ella, Wilson pedía permiso al poeta para incluir su nombre entre los firmantes de un escrito que quería enviar a The Times y distribuir entre los antiguos alumnos y colegas de Trend, con el fin de recaudar fondos para poner en marcha dicha iniciativa bajo la denominación The J. B. Trend Memorial Fund. El texto decía íntegramente lo siguiente:

The work of the late Professor J.B. Trend for Spanish, Portuguese and Latin American studies is well known to scholars and musicians in many countries. His pupils and friends will remember what they owe to his inspiration and personal help. We believe that many would be glad to commemorate him by setting up a fund, associated with his name, to further the studies to which he gave so much. The purpose of this fund would be to enable Cambridge undergraduates and research students to visit Spanish and Portuguese speaking countries in the course of their education in languages, literature, history or music. This is what we think he himself would have wished. ${ }^{66}$

Además de Wilson y de Guillén, que asintió a la petición del anterior, entre los que suscribieron el texto se encontraban Helen Grant, Roberto Gerhard o, por supuesto, Alberto Jiménez Fraud, en lo que se convierte en el resumen de una vida y en el más sincero testimonio de reconocimiento a la labor y la persona del catedrático e hispanista John B. Trend.

Ocho años después, el 30 de julio de 1966, era a Madariaga a quien le llegaba el reconocimiento académico, al ser investido doctor honoris causa por la Universidad de Oxford, su universidad. A raíz del acontecimiento, el periodista John Ezard entrevistaba al profesor en el Oxford Mail y decía de él: "Oxford University, when it gives him his degree, will be paying tribute to one of the most encyclopaedic talents ever to have settled here -a man so broad in his energies that he has been compared to H. G. Wells and George Bernard Shaw" ${ }^{37}$. Acababa de cumplir 80 años y, como Trend, no había pisado la España de Franco; le faltaba, por tanto, el reconocimiento de su país.

Pero este llegó. Porque, al contrario que Trend, Madariaga vivió la caída del dictador y pudo ver de nuevo una España democrática. El 5 de abril de 1976, ya

65 Carta inédita de E.M Wilson a J. Guillén (24 de octubre de 1958). Archivo Jorge Guillén. Biblioteca Nacional de España.

66 "J.B. Trend Memorial". Expediente sobre John B. Trend. Colección Capilla Alfonsina, Instituto Nacional de Bellas Artes, México, D. F. Desde aquí quiero agradecer al profesor Gabriel Rosenzweig su deferencia y generosidad al haberme proporcionado este documento.

67 En Victoria, Vida de Salvador..., p. 397. 
casi nonagenario, regresaba a casa ${ }^{68}$, y un mes más tarde, el 2 de mayo, ingresaba en la Real Academia Española de la Lengua. El nombramiento le había llegado en julio de 1936, pero la guerra no le permitió pronunciar su discurso de ingreso, que iba a versar sobre la Tragicomedia de Calisto y Melibea $^{69}$. Cuatro décadas después, Madariaga varió su argumento y, ante más de ochocientas personas, habló "De la belleza en la ciencia"; quiso empezar su intervención parafraseando el "decíamos ayer" de Fray Luis de León, pero no lo hizo porque llevaba implícito un "nosotros" que apenas ya se encontraba entre los asistentes. A pesar de ello, la Academia, le había reservado su sillón, la letra "M" mayúscula. Julián Marías, en su réplica a Madariaga, así lo hizo ver: "Señores Académicos -afirmó en el emotivo colofón de su discurso-: La Real Academia Española vuelve a estar completa" ${ }^{\text {"70 }}$. 
